\title{
QCD thermodynamics of effective models with an improved Polyakov-loop potential
}

\section{Rainer Stiele, ${ }^{* b}$ Lisa M. Haas, ${ }^{a b}$ Jens Braun, ${ }^{b c}$ Jan M. Pawlowski ${ }^{a b}$ and Jürgen Schaffner-Bielich ${ }^{a b d}$}

a Institut für Theoretische Physik, Universität Heidelberg,

Philosophenweg 16, D-69120 Heidelberg, Germany

${ }^{b}$ ExtreMe Matter Institute EMMI, GSI, Planckstraße 1, D-64291 Darmstadt, Germany

${ }^{c}$ Institut für Kernphysik (Theoriezentrum), Technische Universität Darmstadt,

Schloßgartenstraße 2, D-64289 Darmstadt, Germany

${ }^{d}$ Institut für Theoretische Physik, Goethe-Universität Frankfurt,

Max-von-Laue-Straße 1, D-60438, Frankfurt am Main, Germany

E-mail: R.Stieledthphys.uni-heidelberq.de

L.Haasethphys.uni-heidelberg.de,

Jens.Braunephysik.tu-darmstadt.de,

J.Pawlowskiethphys.uni-heidelberg.de

Schaffnerdastro.uni-frankfurt.de

\begin{abstract}
We analyse the role of the quark backreaction on the gauge-field dynamics and its impact on the Polyakov-loop potential. Based on our analysis we construct an improved Polyakov-loop potential that can be used in future model studies [四]. In the present work, we employe this improved potential in a study of a $2+1$ flavour Polyakov-quark-meson model and show that the temperature dependence of the order parameters and thermodynamics is closer to full QCD. We discuss the results for QCD thermodynamics and outline briefly the dependence of our results on the critical temperature and the parametrisation of the Polyakov-loop potential as well as the mass of the $\sigma$-meson.
\end{abstract}

Xth Quark Confinement and the Hadron Spectrum,

October 8-12, 2012

TUM Campus Garching, Munich, Germany

\footnotetext{
* Speaker.
} 


\section{Introduction}

In low-energy effective models for QCD such as the Polyakov-loop extended Nambu-JonaLasinio (PNJL) and quark-meson (PQM) model the gauge-field dynamics is not fully included. Specifically, the QCD Polyakov-loop potential is approximated by its Yang-Mills (YM) analogue. Therefore, these effective models show some discrepancy in comparison to QCD within functional

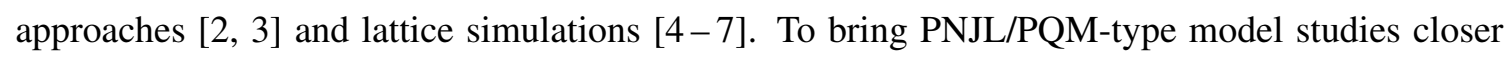
to full QCD, the YM Polyakov-loop potential has to be replaced by the QCD glue potential. The latter is generated by the gauge degrees of freedom in the presence of dynamical quarks, for details see Ref. [四]. We determine the change of the Polyakov-loop potential due to the backreaction of the quarks on the gauge degrees of freedom. We apply this improved Polyakov-loop potential in a 2+1 flavour Polyakov-quark-meson model and compare the results with those obtained with the pure YM potential. A detailed discussion is given in Ref. [四, for related work see Ref. [[8].

\section{The glue potential with functional methods in pure gauge theory and full QCD}

The functional renormalisation group (FRG) equation for the scale dependent quantum effective action, the Wetterich equation, is is depicted in Fig.W for QCD. It provides a setting which allows to approach the low temperature regime from large momentum scales by successively integrating out momentum shells with momenta at about $k$. When lowering the momentum scale one systematically includes quark and gluon fluctuations into the theory finally approaching the hadronic phase. Although the flow equation has a simple one-loop structure, Fig. W describes fully dynamical QCD. In particular, the flow of the gluon propagator receives contributions from matter loops, e.g. the quark contribution to the vacuum polarisation shown in Fig. \.

Evaluated on a general gluonic background $A_{0}$ and integrated over all scales $k$, Fig. W provides the full Polyakov loop potential of QCD in terms of $A_{0}$, see Ref. [[]]. Here the Polyakov loop $\Phi\left[A_{0}\right]$ serves as the confinement-deconfinement order parameter [Q, $[0]$. The simple one loop structure in Fig. Wallows for a straightforward identification of gluonic and matter contributions. In particular, the first two loops in Fig. W generate the glue potential of QCD and depend on the full gluon and ghost propagators in the presence of dynamical quarks. For example, for the gluon this entails

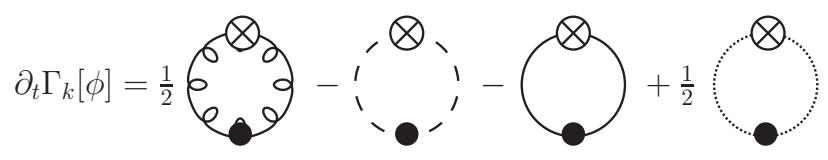

Figure 1: Partially bosonised version of the FRG flow for QCD. The loops denote the gluon, ghost, quark and hadronic contributions, respectively. The crosses mark the $\mathrm{RG}$ regulator term and $t=\log k / \Lambda$ with the infrared momentum scale $k$ and the references scale $\Lambda$.

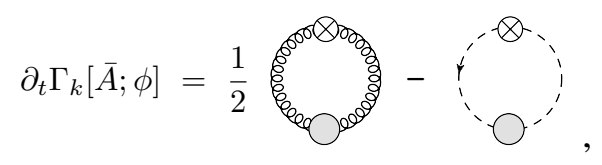

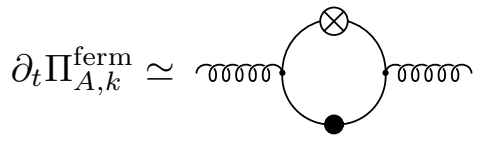

Figure 2: Left: Functional flow for the YM effective action. Right: Quark polarisation contribution to the gluon propagator representing a contribution of the matter backcoupling. 

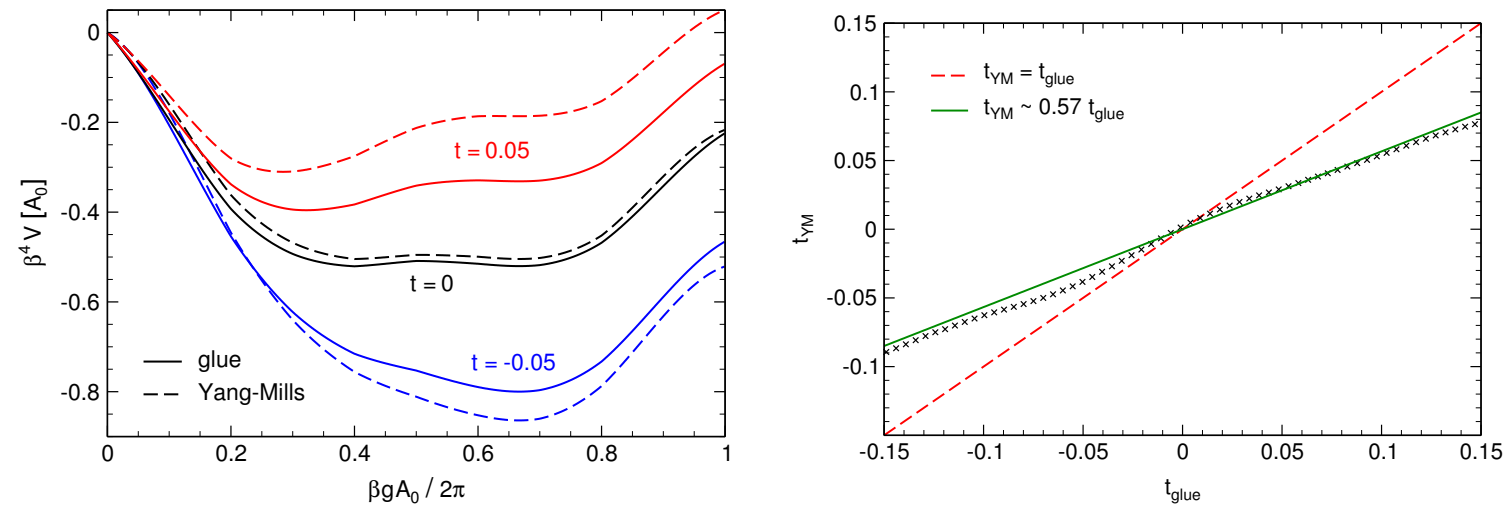

Figure 3: Left: YM potential (dashed lines) and glue effective potential (full lines) as functions of the background gauge field $\left\langle A_{0}\right\rangle$ for various reduced temperatures. The form of the potentials is similar, however the temperature scale changes. Right: Relation between the temperature scales of YM theory and the QCD glue potential including the backreaction of the quarks. We approximate the numerical data (black crosses) by a linear relation (full green line) that clearly deviates from pure YM theory (red dashed line).

the inclusion of the quark part of the vacuum polarisation, see Fig. 口, with fully dynamical quark propagators. In turn, dropping the coupling between matter sector and glue sector, the first two loops in Fig. W simplify to the flow of the glue potential of pure YM theory, see Fig. \. Within the FRG-approach to two flavour QCD detailed in Refs. [Q], []], we have computed the fully dynamical glue potential that takes into account the backreaction of the quark degrees of freedom on the gluon propagators (and vice versa). We compare this potential to the glue potential of SU(3) YM theory as obtained in Refs. [Q, [1, [2]. The first non-trivial result of this comparison is that the $A_{0^{-}}$ dependence of both order-parameter potentials $V\left(\left\langle A_{0}\right\rangle\right)$ agrees on a quantitative level, see Fig. B. This agreement is achieved after a non-trivial matching of the absolute temperature scales and the temperature slope of the potentials. Both effects on the relative temperature dependences are induced by the matter fluctuations altering the ghost and gluon propagators in QCD. We exploit these observations to estimate how the temperature-dependence of a given Polyakov loop model potential for pure YM theory has to be modified to improve it towards a full QCD Polyakov loop potential. As a measure for the distance of the potentials we use

$$
\int_{0}^{2 \pi T / \bar{g}} d\left\langle A_{0}\right\rangle\left|V_{\mathrm{YM}}\left(\left\langle A_{0}\right\rangle\right)-V_{\text {glue }}\left(\left\langle A_{0}\right\rangle\right)\right|^{2} .
$$

In terms of reduced temperatures

$$
t_{\mathrm{glue}}=\left(T-T_{\mathrm{cr}}^{\mathrm{glue}}\right) / T_{\mathrm{cr}}^{\mathrm{glue}} \quad \text { and } \quad t_{\mathrm{YM}}=\left(T-T_{\mathrm{cr}}^{\mathrm{YM}}\right) / T_{\mathrm{cr}}^{\mathrm{YM}}
$$

the minimisation of the measure ([.] $)$ yields the following translation of the two temperature scales

$$
t_{\mathrm{YM}}\left(t_{\text {glue }}\right) \approx 0.57 t_{\text {glue }} .
$$

The critical temperatures of the respective potentials in the FRG computations are $T_{\mathrm{cr}}^{\text {glue }}=203 \mathrm{MeV}$ []] in two-flavour QCD in the chiral limit and $T_{\mathrm{cr}}^{\mathrm{YM}}=276 \mathrm{MeV}$ [Q, 且, [2]. We shall discuss below that the absolute temperature scale still remains a free parameter (within a small interval) in our model. The relation ([2.3) can now serve as an import input for model studies. For the detailed derivation and further details we refer the interested reader to Ref. [四]. 


\section{Polyakov-quark-meson model and Polyakov-loop potential}

Effective models of QCD such as the PNJL [[13]-[6] and the PQM [[7]-[23] model unify aspects of chiral symmetry and confinement. The models are defined via the free energy of QCD as a function of the order parameters and thermodynamic control parameters

$$
\Omega\left(\sigma, \sigma_{\mathrm{s}}, \Phi, \bar{\Phi} ; T, \mu_{f}\right)=U\left(\sigma, \sigma_{\mathrm{s}}\right)+\mathcal{U}(\Phi, \bar{\Phi} ; T)+\Omega_{\mathrm{qq}}\left(\sigma, \sigma_{\mathrm{s}}, \Phi, \bar{\Phi} ; T, \mu_{f}\right)
$$

The particle content of the PQM-model is constituent quarks minimally coupled to gauge fields and coupled to mesons via a Yukawa-type term. The contribution of the mesonic degrees of freedom $U\left(\sigma, \sigma_{\mathrm{s}}\right)$ contains several parameters that are adjusted to meson properties, see e.g. Refs. [24, [25]. The values of the constants that we use to fix these parameters are given in Ref. [W]. A remaining uncertainty in the chiral sector is the mass of the $\sigma$-meson. Within our model we identify it with the resonance $f_{0}(500)$ and we discuss its impact by varying $m_{\sigma}$ in the range of $(400-600) \mathrm{MeV}$. The order parameters of chiral symmetry are the light or non-strange condensate $\sigma$ and the strange chiral condensate $\sigma_{\mathrm{s}}$ that can be combined to the subtracted chiral condensate $\Delta_{\mathrm{l}, \mathrm{s}}$.

For the Polyakov-loop potential $U(\Phi, \bar{\Phi} ; T)$ a functional form is chosen that reproduces the temperature dependence of the Polyakov loop expectation value and the thermodynamics of pure YM theory as obtained in lattice calculations. We compare results obtained with the logarithmic parametrisation of Ref. [ए]] (that we abbreviate with 'Log' in the following) and the polynomial parametrisation with the parameters of Ref. [26] (Poly-I) and those of Ref. [14]] (Poly-II). We use our relation between the pure gauge and the glue effective potential (2.3) to effectively convert these pure gauge potentials to the glue potential of full QCD

$$
\mathcal{U}_{\text {glue }}\left(\Phi, \bar{\Phi}, t_{\text {glue }}\right) / T^{4}=\mathcal{U}_{\mathrm{YM}}\left(\Phi, \bar{\Phi}, t_{\mathrm{YM}}\right) / T_{\mathrm{YM}}^{4} \cdot
$$

The absolute temperature scale for the case of physical quark masses (pion masses) has not been computed in Ref. [[]]. Therefore, we consider the glue critical temperature in the model as a free parameter that we vary in the range $180 \mathrm{MeV} \lesssim T_{\mathrm{cr}}^{\text {glue }} \lesssim 270 \mathrm{MeV}$ [प]], for details see Ref. [四].

The last term of Eq. (B.]) represents the quark sector that includes the coupling to the Polyakovloop variables and the mesons. For a detailed discussion of the $2+1$ flavour model we refer to Refs. [1, [18, [2]].

\section{Results and discussion}

The temperature dependence of the thermodynamics and the order parameters displayed in Figs. $\rightarrow$ and $\$$ shows that replacing the pure YM Polyakov-loop potential by the quark-improved potential leads to a significantly smoother crossover transition at vanishing density. The pressure is in remarkable agreement with the result from lattice calculations given the mean field nature of the matter sector. The amplitude of the trace anomaly or interaction measure follows the lattice calculations as well. We observe that the behaviour of the pressure and interaction measure in the transition region is still slightly steeper. Also the evolution of the chiral order parameter shows a smoother evolution when applying the quark-improved Polyakov-loop potential. Including fluctuations will bring the smoothening towards the lattice result as will be discussed in a forthcoming 

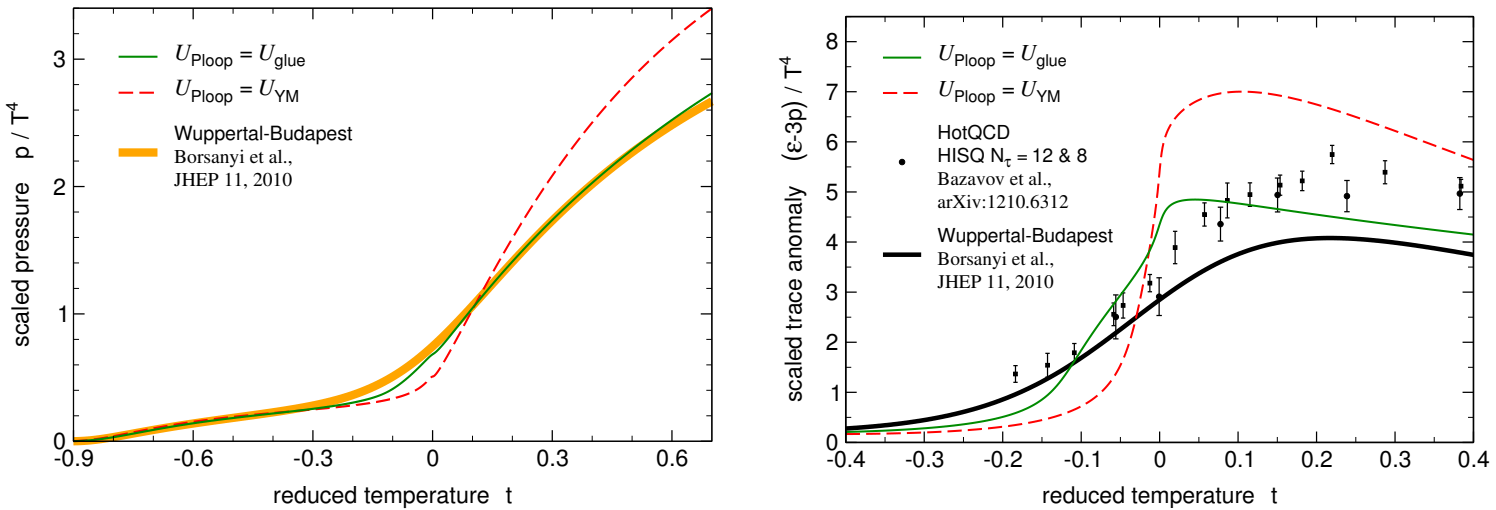

Figure 4: Normalised pressure (left) and trace anomaly or interaction measure (right) approximating the Polyakov-loop potential with the pure gauge potential (red dashed lines) and with the quark-improved glue

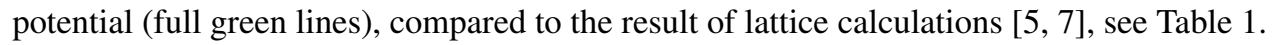
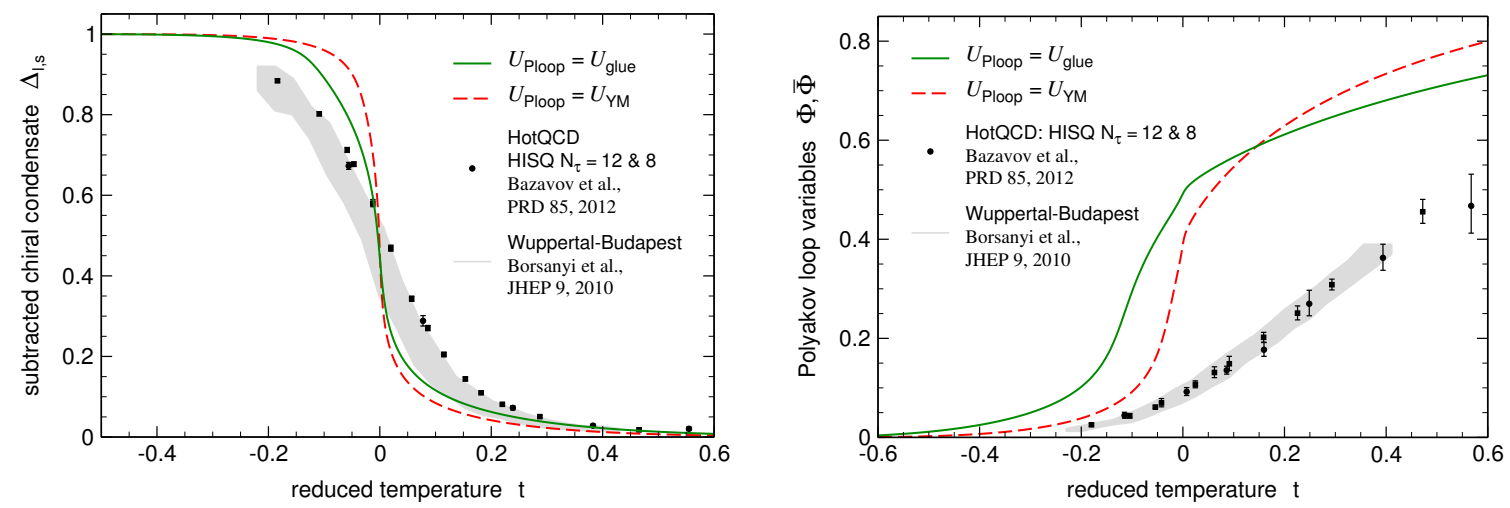

Figure 5: Subtracted chiral condensate (left) and Polyakov loop (right) approximating the Polyakov-loop potential with the pure gauge potential (red dashed lines) and with the quark-improved glue potential (full

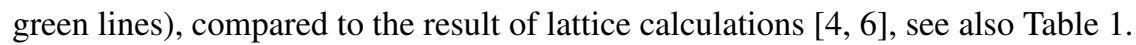

\begin{tabular}{c|c|c|c|c} 
& $\mathcal{U}_{\mathrm{YM}}$ & $\mathcal{U}_{\text {glue }}$ & Wuppertal-Budapest [四] & HotQCD $N_{\tau}=12 \& 8[$ [ $]$ \\
\hline$T_{\mathrm{c}}[\mathrm{MeV}]$ & 168 & 158 & 157 & $159 \& 163$
\end{tabular}

Table 1: Pseudocritical temperatures for the crossover phase transition at $\mu_{f}=0$. They are determined by the peaks in the temperature derivatives of the subtracted condensate $\Delta_{1, \mathrm{~s}}$. For the effective model calculations we use the logarithmic parametrisation of the Polyakov-loop potential with a critical temperature of $210 \mathrm{MeV}$ and a sigma meson mass of $500 \mathrm{MeV}$.

publication. The pseudocritical temperatures summarised in Table $\mathbb{W}$ show that the result with the quark-improved Polyakov-loop potential agrees well with the value found in lattice calculations. The result for the Polyakov loop is shifted to higher values in the confined phase and to lower values in the deconfined phase when replacing the YM Polyakov-loop potential by the effective glue potential, smoothening the transition. This is a consequence of applying Eq. (ㄹ.3]) as can be also seen in Fig. [1. The remaining difference can be attributed to the missing quark-meson dynamics and that the Polyakov loop potential is written in terms of the variable $\left\langle\Phi\left[A_{0}\right]\right\rangle$ while the coupling to the matter sector is described as a function of $\Phi\left[\left\langle A_{0}\right\rangle\right]$, see the discussion in Refs. [U, [1], [2]]. 

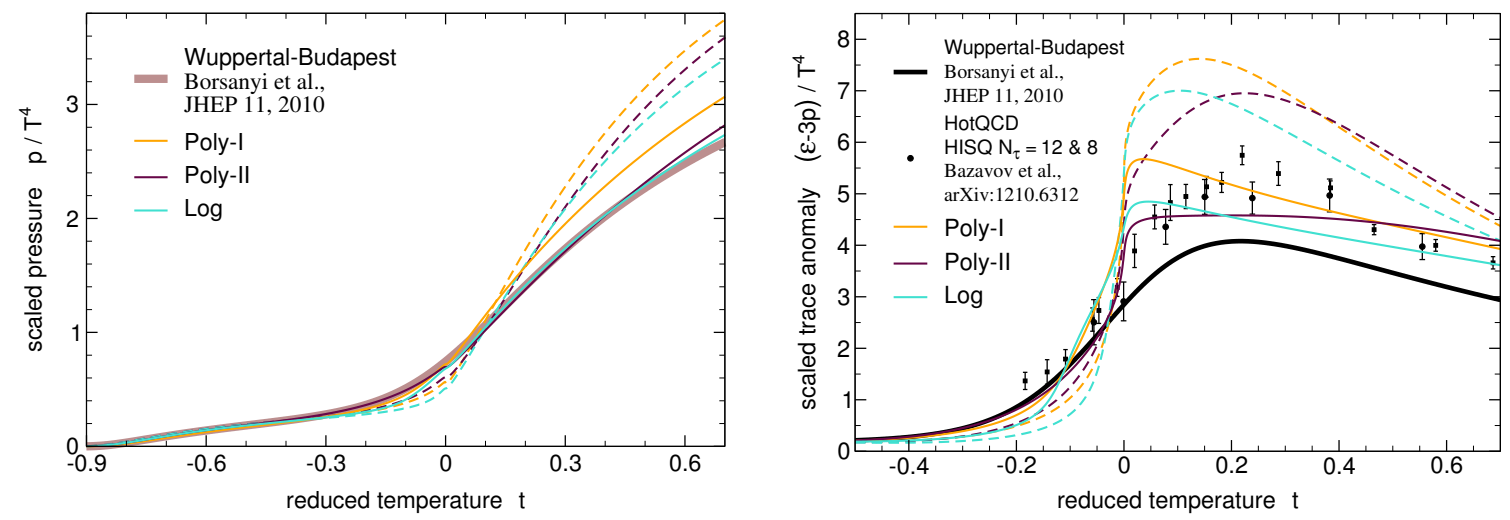

Figure 6: Normalised pressure (left) and trace anomaly or interaction measure (right) for different parametri-

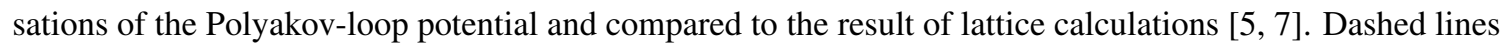
stand for the case of approximating the Polyakov-loop potential with the YM potential and solid lines are for the case of using the improved glue potential. We use a transition temperature of the Polyakov-loop potential of $210 \mathrm{MeV}$ and a sigma meson mass of $500 \mathrm{MeV}$.

\begin{tabular}{c|c|c|c} 
& Poly-I & Poly-II & Log \\
\hline $\mathcal{U}_{\text {YM }}$ & 158 & 169 & 168 \\
$\mathcal{U}_{\text {glue }}$ & 144 & 158 & 158
\end{tabular}

\begin{tabular}{c|c|c|c|c}
$T_{\text {cr }}^{\text {glue }}[\mathrm{MeV}]$ & 180 & 210 & 240 & 270 \\
\hline $\mathcal{U}_{\text {YM }}$ & 156 & 168 & 183 & 198 \\
$\mathcal{U}_{\text {glue }}$ & 152 & 158 & 164 & 171
\end{tabular}

Table 2: Pseudocritical temperatures in $\mathrm{MeV}$ for the crossover transition at $\mu_{f}=0$. Left: For different parametrisations and parameter sets of the Polyakov-loop potential using a transition temperature of the potential of $210 \mathrm{MeV}$. Right: For different critical temperatures of the Polyakov-loop potential using the logarithmic parametrisation. For both cases we use a sigma meson mass of $500 \mathrm{MeV}$. The pseudocritical temperatures are determined by the peaks of the chiral susceptibility $\partial \Delta_{\mathrm{l}, \mathrm{s}} / \partial T$.

The uncertainties within our model study are the parametrisation and the transition temperature of the Polyakov-loop potential and the mass of the $\sigma$-meson. Figure 6 shows the sensitivity on the parametrisation and different parameter sets of the glue sector. The results within the different descriptions of the YM or glue potential are compatible but the transition is in general smoother with the quark-improved glue potential. The amplitude of the trace anomaly is in better agreement with lattice calculations. Note, however, that the temperature dependence is slightly steeper independent of the actual parametrisation. The maximum of the interaction measure above $T_{\mathrm{c}}$ with the Poly-II YM Polyakov-loop potential corresponds to the nearly constant behaviour above $T_{\mathrm{c}}$ when the glue Polyakov-loop potential is applied. Table $\square$ summarises the pseudocritical temperatures for the different calculations. Both the steepness as well as the flat behaviour above $T_{c}$ are expected shortcomings of the present mean-field nature of the matter dynamics. The effects of including a fully dynamical matter sector will be discussed in a forthcoming publication.

Our analysis of the impact of the transition temperature $T_{\mathrm{cr}}^{\mathrm{glue}}$ of the Polyakov-loop potential on thermodynamic observables shown in Fig. $\square$ confirms that the temperature dependence of the pressure and the interaction measure is in far better agreement with lattice calculations when the quark-improved Polyakov-loop potential is used. The transition gets steeper when the transition temperature of the glue potential is decreased. We find best agreement with lattice calculations for $T_{\mathrm{cr}}^{\text {glue }} \sim 210 \mathrm{MeV}$ but this scale could be lowered by the inclusion of fluctuations [23]. The 

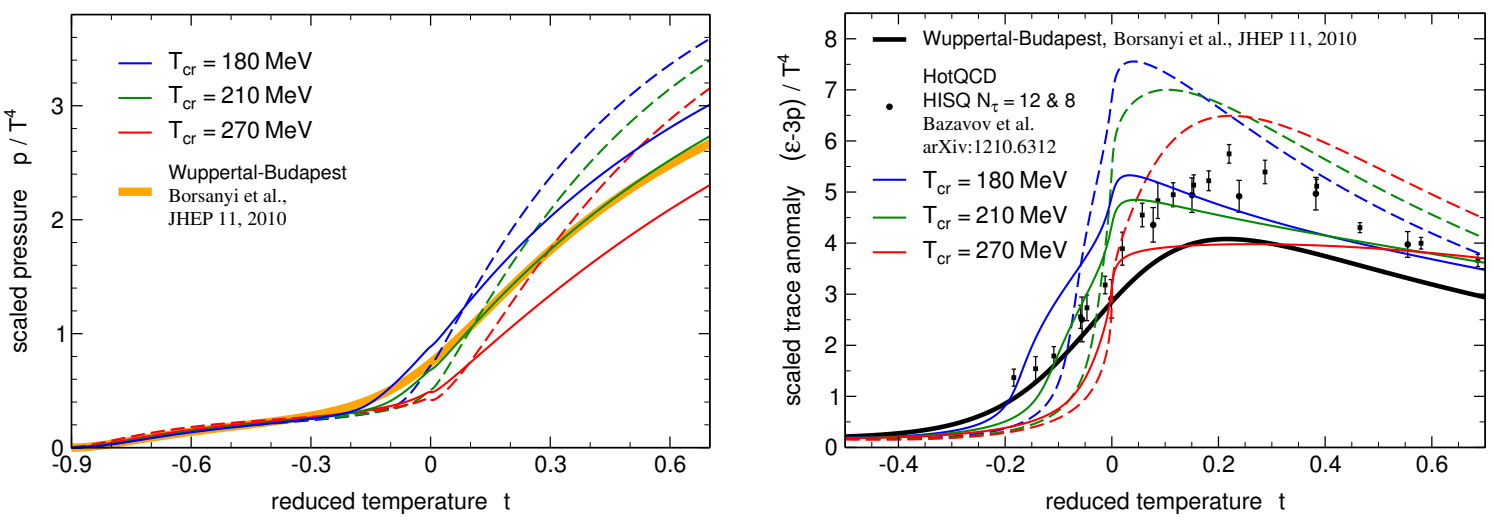

Figure 7: Normalised pressure (left) and trace anomaly or interaction measure (right) for different transition temperatures of the Polyakov-loop potential compared to the result of lattice calculations [ [ [, प]. Dashed lines are for the YM potential case and solid lines are for the improved glue potential. The logarithmic parametrisation of the Polyakov-loop potential and a sigma meson mass of $500 \mathrm{MeV}$ is used.
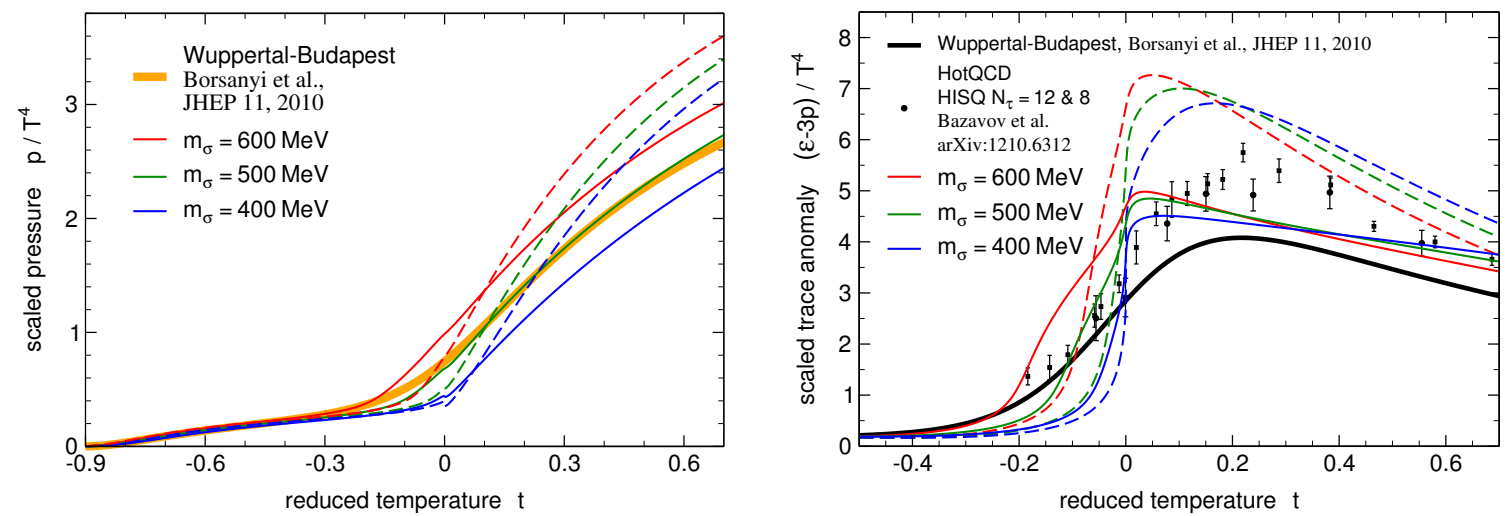

Figure 8: Same as the previous figure for different masses of the scalar sigma meson. The logarithmic parametrisation of the Polyakov-loop potential and a critical temperature of $210 \mathrm{MeV}$ is used.

temperature dependence of the trace anomaly shows that the transition region expands below the pseudocritical temperature if the transition temperature $T_{\mathrm{cr}}^{\mathrm{glue}}$ of the glue potential is decreased. The pseudocritical temperature is at the lower end of the transition region for larger critical temperatures of the Polyakov-loop potential. The absolute values of the pseudocritical temperatures are given in Table ․

The dependence of the results on the mass of the $\sigma$-meson (Fig. [8]) shows that the pseudocritical temperature moves from the lower end of the transition region to the upper end when the $\sigma$-meson mass is increased. Increasing $m_{\sigma}$ has an opposite effect to increasing the transition temperature of the Polyakov-loop potential. The quantitative best agreement with the pressure of lattice calculations is obtained for the combinations $\left(m_{\sigma}, T_{\mathrm{cr}}^{\mathrm{glue}}\right)$ of the $\sigma$-meson mass and the Polyakovloop potential transition temperature of $(400 \mathrm{MeV}, 180 \mathrm{MeV}),(500 \mathrm{MeV}, 210 \mathrm{MeV})$ and $(600 \mathrm{MeV}$, $250 \mathrm{MeV})$. The best agreement with lattice QCD data for the pseudocritical temperature is found for the combination $m_{\sigma}=500 \mathrm{MeV}$ and $T_{\mathrm{cr}}^{\text {glue }}=210 \mathrm{MeV}$, as can be read off from Table Bl. 


\begin{tabular}{c|c|c|c}
$m_{\sigma}[\mathrm{MeV}]$ & 400 & 500 & 600 \\
\hline $\mathcal{U}_{\text {YM }}$ & 161 & 168 & 179 \\
$\mathcal{U}_{\text {glue }}$ & 144 & 158 & 173
\end{tabular}

Table 3: Pseudocritical temperatures for the crossover transition at $\mu_{f}=0$ for different masses of the sigma meson. They are determined by the peaks in the temperature derivatives of the subtracted condensate $\Delta_{\mathrm{l}, \mathrm{s}}$. We use the logarithmic parametrisation of the Polyakov-loop potential with a critical temperature of $210 \mathrm{MeV}$.

\section{Acknowledgments}

We thank L. Fister, E. S. Fraga, T. K. Herbst, B. W. Mintz and B.-J. Schaefer for discussions and collaboration on related topics. This work is supported by BMBF under grants FKZ 05P12VHCTG and 06HD7142, by ERC-AdG-290623, by the Helmholtz Alliance HA216/EMMI, by the DFG through the HGSFP, by HGS-HIRe, and by HIC for FAIR within the LOEWE program.

\section{References}

[1] L. M. Haas, R. Stiele, J. Braun, J. M. Pawlowski, and J. Schaffner-Bielich, GrXiv:1302.1993 [hep-ph].

[2] J. Braun, L. M. Haas, F. Marhauser, and J. M. Pawlowski, Phys. Rev. Lett. $106(2011) 022002$, arXiv:0908.0008 [hep-ph].

[3] J. M. Pawlowski, AIP Conf. Proc. 1343 (2011) 75-80, arXiv:1012.5075 [hep-ph]

[4] Wuppertal-Budapest Collaboration, S. Borsanyi et al., VHEP 1009 (2010) 073, arXiv: 1005.3508 hep-lat].

[5] Wuppertal-Budapest Collaboration, S. Borsanyi et al., THEP 1011 (2010) 077, ErXiv: 1007.2580 hep-lat].

[6] HotQCD Collaboration, A. Bazavov et al., Phys. Rev. D 85 (2012) 054503, arxiv: 1111.1710 [hep-Iat.

[7] HotQCD Collaboration, A. Bazavov, Nucl. Phys. A (2012), arXiv:1210.6312 [hep-lat].

[8] K. Fukushima and K. Kashiwa, arXiv:1206.0685 [hep-ph]].

[9] J. Braun, H. Gies, and J. M. Pawlowski, Phys. Lett. B 684 (2010) 262-267, arXiv:0708.2413 thep-th.

[10] F. Marhauser and J. M. Pawlowski, arXiv:0812.1144 hep-ph]

[11] J. Braun, A. Eichhorn, H. Gies, and J. M. Pawlowski, Eur. Phys. J. C70 (2010) 689-702 arXiv:1007.2619 hep-ph].

[12] L. Fister and J. M. Pawlowski, arXiv:1301.4163 [hep-ph].

[13] K. Fukushima, Phys. Lett. B 591 (2004) 277-284, arXiv: hep-ph/0310121 hep-ph].

[14] C. Ratti, M. A. Thaler, and W. Weise, Phys. Rev. D 73 (2006) 014019, arXiv: hep-ph/0506234 एhep-ph.

[15] E. Megias, E. Ruiz Arriola, and L. Salcedo, Phys. Rev. D 74 (2006) 065005, arXiv:hep-ph/0412308 [hep-ph]. 
[16] S. Roessner, C. Ratti, and W. Weise, Phys. Rev. D 75 (2007) 034007, arXiv:hep-ph/0609281 [hep-ph].

[17] B.-J. Schaefer, J. M. Pawlowski, and J. Wambach, Phys. Rev. D 76 (2007) 074023, arXiv:0704.3234 [hep-ph].

[18] B.-J. Schaefer, M. Wagner, and J. Wambach, Phys. Rev. D 81 (2010) 074013, GrXiv:0910.5628 thep-ph].

[19] V. Skokov, B. Stokic, B. Friman, and K. Redlich, Phys. Rev. C 82 (2010)015206, arXiv:1004.2665 [hep-ph].

[20] T. K. Herbst, J. M. Pawlowski, and B.-J. Schaefer, Phys. Lett. B 696 (2011) 58-67, arXiv:1008.0081 [hep-ph].

[21] B. Schaefer and M. Wagner, Phys. Rev. D 85 (2012) 034027, arXiv:1111.6871 hep-ph]

[22] B. W. Mintz, R. Stiele, R. O. Ramos, and J. Schaffner-Bielich, Phys. Rev. D 87 (2013) 036004, arXiv: 1212.1184 hep-ph].

[23] T. K. Herbst, J. M. Pawlowski, and B.-J. Schaefer, arXiv:1302.1426 [hep-ph].

[24] J. T. Lenaghan, D. H. Rischke, and J. Schaffner-Bielich, Phys. Rev. D 62 (2000) 085008, arXiv:nucl-th/0004006 nucl-th].

[25] B.-J. Schaefer and M. Wagner, Phys. Rev. D 79 (2009) 014018, arXiv:0808.1491 hep-ph].

[26] O. Scavenius, A. Dumitru, and J. Lenaghan, Phys. Rev. C 66 (2002) 034903, arXiv:hep-ph/0201079 hep-ph].

[27] J. Braun and A. Janot, Phys. Rev. D 84(2011) 114022, arXiv:1102.4841 [hep-ph]. 